\title{
Numerical Computation for a Kind of Time Optimal Control Problem for the Tubular Reactor System
}

\author{
Detang Zeng $\mathbb{D}^{1},{ }^{1,2}$ Xin Yu, ${ }^{3}$ Jingfang Huang, ${ }^{4}$ and Chunqing Tan ${ }^{1,2}$ \\ ${ }^{1}$ Institute of Engineering Thermophysics, Chinese Academy of Science, Beijing 100190, China \\ ${ }^{2}$ University of Chinese Academy of Sciences, Beijing 100049, China \\ ${ }^{3}$ Laboratory of Information \& Control Technology, Ningbo Institute of Technology, Zhejiang University, Ningbo 315100, China \\ ${ }^{4}$ School of Mathematical Sciences, Zhejiang University, Hangzhou 310027, China
}

Correspondence should be addressed to Detang Zeng; zengdetang@iet.cn

Received 14 December 2017; Accepted 26 February 2018; Published 4 April 2018

Academic Editor: Thierry Floquet

Copyright (c) 2018 Detang Zeng et al. This is an open access article distributed under the Creative Commons Attribution License, which permits unrestricted use, distribution, and reproduction in any medium, provided the original work is properly cited.

\begin{abstract}
This paper is devoted to the study of numerical computation for a kind of time optimal control problem for the tubular reactor system. This kind of time optimal control problem is aimed at delaying the initiation time $\tau$ of the active control as late as possible, such that the state governed by this controlled system can reach the target set at a given ending time $T$. To compute the time optimal control problem, we firstly approximate the original problem by finite element method and get a new approximation time optimal control problem governed by ordinary differential equations. Then, through the control parameterization method and time-scaling transformation, the approximation problem becomes an optimal parameter selection problem. Finally, we use Sequential Quadratic Program algorithm to solve the optimal parameter selection problem. A numerical simulation is given for illustration.
\end{abstract}

\section{Introduction}

Tubular reactors have extensive applications in the industrial production. A lot of reaction processes in chemical and biochemical engineering can be described by tubular reactor models, such as chlorine dioxide bleaching model [1]. The dynamic tubular reactors are typically described by nonlinear partial differential equations (PDEs), which include convection, reaction, and diffusion phenomena. For studies of such PDEs system, we would mention some works $[2,3]$. Sometimes we regulate the reaction process artificially to improve the reactor rate or reduce the reaction time, by seeking some control strategies such as adding catalyst or placing a heating or cooling jacket. For this purpose, optimal control problems governed by such PDEs system arouse increasing attentions [4-7]. As a kind of important optimal control problem, time optimal control problem plays an important role in many fields of applications.

Generally speaking, time optimal control problems can be divided into two types. The first one is to find a minimal time $T$ and a control belonging to some constraint set which is acted upon from beginning time 0 , such that the state governed by the controlled system can arrive at a given target set in the shortest time interval $[0, T]$. This kind of problem is called the minimal time optimal control problem. The second one is to delay the initiation time $\tau$ of the active control as late as possible, such that the state governed by this controlled system can reach the target set at the given ending time $T$. This is a maximal time optimal control problem. There have been extensive researches on the first kind of time optimal control problem [8-11], but only a few works related to the second kind of time optimal control problem have been studied. For the second kind of time optimal control problem, we would mention the works $[12,13]$.

In this paper, we shall consider the second kind of time optimal control problem with boundary controls governed by a coupled tubular reactor system, which is described by nonlinear partial differential equations and corresponds to the following chemical reaction process $[14,15]$ :

$$
R_{1}+R_{2} \longrightarrow P
$$




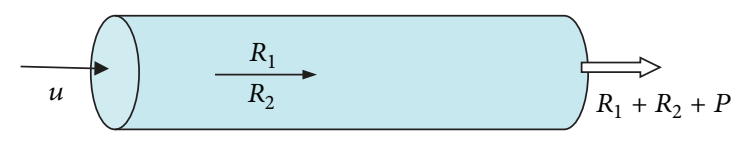

FIgURE 1: Tubular reactor.

where $R_{1}, R_{2}$ are two reactants and $P$ is the product of the chemical reaction. We assume that the kinetics depend on the concentrations of two reactants $R_{1}$ and $R_{2}$, which has the form $\left(-r_{1} x y,-r_{2} x y\right)^{\top}$, where $r_{i}, i=1,2$, are the kinetic constants and $x, y$ are the concentrations of the reactants $R_{1}$ and $R_{2}$, respectively. Moreover, we assume that the length of the reactor is $l$. A control $u$ is acted upon on the one side of the tubular reactor to control the reactor process, as depicted in Figure 1. More precisely, the above controlled process can be described as follows:

$$
\begin{aligned}
x_{t}(z, t)= & \beta_{1} x_{z z}(z, t)-v x_{z}(z, t) \\
& -r_{1} x(z, t) y(z, t), \\
\qquad 0<z<l, 0<t \leq T, & \\
y_{t}(z, t)= & \beta_{2} y_{z z}(z, t)-v y_{z}(z, t) \\
& -r_{2} x(z, t) y(z, t), \\
& 0<z<l, 0<t \leq T,
\end{aligned}
$$

$$
\begin{aligned}
\beta_{1} x_{z}(0, t) & =u_{1}(t), \\
x_{z}(l, t) & =0
\end{aligned}
$$

$$
0<t \leq T
$$

$$
\begin{aligned}
\beta_{2} y_{z}(0, t) & =u_{2}(t), \\
y_{z}(l, t) & =0
\end{aligned}
$$

$$
0<t \leq T
$$

$$
\begin{aligned}
& x(z, 0)=x_{0}(z), \\
& y(z, 0)=y_{0}(z),
\end{aligned}
$$

where $x_{0}, y_{0} \in L^{2}(0, l)$ are the given initial data, $T$ is a given positive number, $\beta_{1}, \beta_{2}$ are the dispersion coefficients, $v$ is the superficial fluid velocity, and $u=\left(u_{1}, u_{2}\right)^{\top} \in$ $L^{2}(0, T) \times L^{2}(0, T)$ is the boundary control. We denote by $\left(x\left(z, t ; u, x_{0}\right), y\left(z, t ; u, y_{0}\right)\right)^{\top}$ the solution to (2) corresponding to the control $u$ and the initial data $\left(x_{0}, y_{0}\right)^{\top}$.

Let $a_{i}, b_{i} \in \mathbb{R}$ for $i=1,2$. Define

$$
\begin{aligned}
S & =\left\{\left(x_{1}, x_{2}\right)^{\top} \mid a_{i} \leq x_{i} \leq b_{i}, i=1,2\right\}, \\
\mathcal{U} & =\left\{(u, \tau) \mid u \in L^{2}(0, T) \times L^{2}(0, T), \tau\right. \\
& \in[0, T], u(t)=0 \text { for a.e. } t \in[0, \tau), u(t) \\
& \in S \text { for a.e. } t \in[\tau, T]\} .
\end{aligned}
$$

Let $\alpha_{i}, \gamma_{i} \in \mathbb{R}$ for $i=1,2$. Define

$$
\begin{aligned}
\mathscr{K} & =\left\{\left(y_{1}, y_{2}\right)^{\top} \in L^{2}(0, l) \times L^{2}(0, l) \mid \alpha_{i} \leq y_{i}(z)\right. \\
& \left.\leq \gamma_{i} \text { for a.e. } z \in[0, l], i=1,2\right\} .
\end{aligned}
$$

The second kind of time optimal control problem governed by (2) can be stated as follows:

$$
\max _{(u, \tau) \in \mathscr{U}}\left\{\tau \mid\left(x\left(z, T ; u, x_{0}\right), y\left(z, T ; u, y_{0}\right)\right)^{\top} \in \mathscr{K}\right\},
$$

where $\left(x\left(z, t ; u, x_{0}\right), y\left(z, t ; u, y_{0}\right)\right)^{\top}$ is the solution to (2). We assume that, for given $\left(x_{0}, y_{0}\right)^{\top} \in L^{2}(0, l) \times L^{2}(0, l)$, there exists $(u, \tau) \in \mathscr{U}$, such that

$$
\left(x\left(\cdot, T ; u, x_{0}\right), y\left(\cdot, T ; u, y_{0}\right)\right)^{\top} \in \mathscr{K} .
$$

$u=\left(u_{1}, u_{2}\right)^{\top} \in L^{2}(0, T) \times L^{2}(0, T)$ satisfying the above assumption is called an admissible control for the problem $(\mathscr{T} \mathscr{P})$. Obviously, the above assumption implies that the admissible control set is not empty. In the problem $(\mathscr{T} \mathscr{P})$, $\tau$ represents the initiation time of the active control. We call

$$
\tau^{*}=\max _{(u, \tau) \in \mathscr{U}}\left\{\tau \mid\left(x\left(z, T ; u, x_{0}\right), y\left(z, T ; u, y_{0}\right)\right)^{\top} \in \mathscr{K}\right\}
$$

the optimal time for the problem $(\mathscr{T} \mathscr{P})$, and a control $u^{*}=\left(u_{1}^{*}, u_{2}^{*}\right)^{\top}$ satisfying $\left(u^{*}, \tau^{*}\right) \in \mathcal{U}$ such that $(x(z, T$; $\left.\left.u^{*}, x_{0}\right), y\left(z, T ; u^{*}, y_{0}\right)\right)^{\top} \in \mathscr{K}$ is called an optimal control for the problem $(\mathscr{T} \mathscr{P})$. The goal of this problem is to find a maximal $\tau^{*}$ and a control $u^{*}$ such that $\left(u^{*}, \tau^{*}\right) \in \mathcal{U}$, and the state of (2) driven by this control can reach the given target set $\mathscr{K}$ at $T$ with the shortest actively controlled interval $\left[\tau^{*}, T\right]$.

The time optimal control problems governed by PDEs are infinite dimensional control problems and are difficult in finding out analytical solutions. Thus, the numerical approximations of time optimal control problems attract a lot of attentions. Here, we would like to mention some related works [16-18] on the computations of time optimal control problems. However, the works mentioned above focused on the first kind of time optimal control problem. To the best of our knowledge, it seems that no attempts have been made to develop the computation for the second kind of time optimal control problem governed by nonlinear PDEs systems. In this paper, we shall consider the computation for the second kind of time optimal control problem $(\mathscr{T} \mathscr{P})$. Firstly, we project the time optimal control problem $(\mathscr{T} \mathscr{P})$ by finite element method into an approximation problem $\left(\mathscr{T} \mathscr{P}^{h}\right)$. Although approximation problem $\left(\mathscr{T} \mathscr{P}^{h}\right)$ is governed by ordinary differential equations, it is difficult to solve directly due to the unknown time variables. Then, we translate problem $\left(\mathscr{T} \mathscr{P}^{h}\right)$ to an optimal parameter selection problem $\left(\mathscr{T} \mathscr{P}_{p}^{h}\right)$ through the control parameterization method and timescaling transformation. Finally, we use Sequential Quadratic Program (SQP) algorithm to solve the optimal parameter selection problem.

The rest of this paper is organized as follows. In Section 2, we give the finite element approximation for the time optimal control problem $(\mathscr{T} \mathscr{P})$. In Section 3, we provide the control parameter method and time-scaling transformation to 
reduce the approximation problem to an optimal parameter selection problem. In Section 4, we give the procedure of solving the optimal parameter selection problem. In Section 5, we carry out some numerical experiments to illustrate the effectiveness of our approximation method.

\section{The Finite Element Approximation of the Problem $(\mathscr{T} \mathscr{P})$}

In this section, we describe finite element approximation of the problem $(\mathscr{T} \mathscr{P})$. Firstly, we introduce a standard triangulation $\mathscr{T}^{h}$ of $[0, l]$. We decompose $[0, l]$ into finite number of subintervals $\left[z_{j-1}, z_{j}\right], j=1,2, \ldots, N$, where $N$ is a positive integer and $z_{j}, j=0,1, \ldots, N$, are grid points which satisfy

$$
0=z_{0}<z_{1}<\cdots<z_{N-1}<z_{N}=l .
$$

$h=\max \left\{z_{j}-z_{j-1} \mid j=1, \ldots, N\right\}$ is called the mesh size of the triangulation $\mathscr{T}^{h}$. Thus, we can write $\mathscr{T}^{h}$ as

$$
\mathscr{T}^{h}=\left\{I_{j}=\left[z_{j-1}, z_{j}\right] \mid j=1,2, \ldots, N\right\} .
$$

Corresponding to each triangulation $\mathscr{T}^{h}$, we can define a finite dimensional space as follows:

$$
\begin{aligned}
S^{h} & =\left\{w_{h} \in C[0, l]\left|w_{h}\right|_{I_{j}} \in P_{1}\left(I_{j}\right) \text { for } j\right. \\
& =1,2, \ldots, N\},
\end{aligned}
$$

where $P_{1}\left(I_{j}\right)$ stands for the space of all linear polynomials defined on the subinterval $I_{j}$. Obviously, the basis of $S^{h}$ can be taken as $\left\{e_{i} \mid i=0,1, \ldots, N\right\}$, where $e_{i} \in S^{h}, i=0,1, \ldots, N$, are continuous and piecewise linear polynomials that satisfy

$$
e_{i}\left(z_{j}\right)= \begin{cases}1, & i=j \\ 0, & i \neq j\end{cases}
$$

Thus, it is clear that $S^{h}$ is a space of $N+1$ dimensions and $S^{h} \subset H^{1}(0, l)$.

Next we construct the approximation of (2). Let $P^{h}$ be the $L^{2}$-projection from $L^{2}(0, l)$ onto $S^{h}$, defined by

$$
\left(P^{h} v, w_{h}\right)=\left(v, w_{h}\right), \quad \forall v \in L^{2}(0, l), w_{h} \in S^{h} .
$$

Here and in what follows, $(\cdot, \cdot)$ denotes the usual inner product of $L^{2}(0, l)$. Moreover, if there is no risk to make any confusion, we will use $x(t)$ to denote a function $x(\cdot, t) \epsilon$ $L^{2}(0, l)$ for a.e. $t \in[0, T]$. Then, the finite element approximation of (2) can be defined as follows: seek functions $x_{h}$ and $y_{h}$ satisfying that $x_{h}(t) \in S^{h}$ and $y_{h}(t) \in S^{h}$ for a.e. $t \in[0, T]$, such that

$$
\begin{aligned}
& \left(\partial_{t} x_{h}(t), w_{h}\right)+\beta_{1}\left(\partial_{z} x_{h}(t), \partial_{z} w_{h}\right)+u_{1}(t) w_{h}(0) \\
& +v\left(\partial_{z} x_{h}(t), w_{h}\right)+\left(r_{1} x_{h}(t) y_{h}(t), w_{h}\right)=0, \\
& \forall w_{h} \in S^{h}, \\
& \left(\partial_{t} y_{h}(t), w_{h}\right)+\beta_{2}\left(\partial_{z} y_{h}(t), \partial_{z} w_{h}\right)+u_{2}(t) w_{h}(0) \\
& +v\left(\partial_{z} y_{h}(t), w_{h}\right)+\left(r_{2} x_{h}(t) y_{h}(t), w_{h}\right)=0, \\
& \forall w_{h} \in S^{h}, \\
& x_{h}(0)=P^{h} x_{0}, \\
& y_{h}(0)=P^{h} y_{0}
\end{aligned}
$$

Noting that $x_{h}(t), y_{h}(t) \in S^{h}$, we can write

$$
\begin{aligned}
& x_{h}(z, t)=\sum_{j=0}^{N} X_{j}(t) e_{j}(z), \\
& y_{h}(z, t)=\sum_{j=0}^{N} Y_{j}(t) e_{j}(z) .
\end{aligned}
$$

The initial conditions can be written as

$$
\begin{aligned}
& x_{h}(0)=P^{h} x_{0}(z)=\sum_{j=0}^{N} X_{j}(0) e_{j}(z), \\
& y_{h}(0)=P^{h} y_{0}(z)=\sum_{j=0}^{N} Y_{j}(0) e_{j}(z) .
\end{aligned}
$$

Substituting (13) into (12) and taking $w_{h}=e_{i}, i=0,1, \ldots, N$, we can obtain that

$$
\begin{aligned}
& \sum_{j=0}^{N}\left(e_{i}, e_{j}\right) \dot{X}_{j}(t)+\beta_{1} \sum_{j=0}^{N}\left(e_{i}^{\prime}, e_{j}^{\prime}\right) X_{j}(t) \\
& \quad+\sum_{j=0}^{N} v\left(e_{i}, e_{j}^{\prime}\right) X_{j}(t)+u_{1}(t) e_{i}(0) \\
& \quad+r_{1} \sum_{j=0}^{N} \sum_{k=0}^{N} X_{j}(t) Y_{k}(t)\left(e_{j} e_{k}, e_{i}\right)=0, \\
& \quad+\sum_{j=0}^{N} v\left(e_{i}, e_{j}^{\prime}\right) Y_{j}(t)+u_{2}(t) e_{i}(0) \\
& \quad+r_{2} \sum_{j=0}^{N} \sum_{k=0}^{N} X_{j}(t) Y_{k}(t)\left(e_{j} e_{k}, e_{i}\right)=0, \\
& \quad+\beta_{2} \sum_{j=0}^{N}\left(e_{i}^{\prime}, e_{j}^{\prime}\right) Y_{j}(t) \\
& \quad i=0,1, \ldots, N, \ldots, N,
\end{aligned}
$$


where $e_{j}^{\prime}$ denotes the derivative of $e_{j}$ with respect to the spacial variable $z$ and $\dot{X}_{j}$ and $\dot{Y}_{j}$ denote the derivatives of $X_{j}$ and $Y_{j}$ with respect to the time variable $t$, respectively. Moreover, it follows from (11) and (14) that

$$
\begin{aligned}
& \sum_{j=0}^{N}\left(e_{i}, e_{j}\right) X_{j}(0)=\left(x_{0}, e_{i}\right), \quad i=0,1, \ldots, N, \\
& \sum_{j=0}^{N}\left(e_{i}, e_{j}\right) Y_{j}(0)=\left(y_{0}, e_{i}\right), \quad i=0,1, \ldots, N .
\end{aligned}
$$

To simplify the notation, let

$$
\begin{aligned}
& X(t)=\left[X_{j}(t)\right]_{(N+1) \times 1} \in \mathbb{R}^{N+1}, \\
& Y(t)=\left[Y_{j}(t)\right]_{(N+1) \times 1} \in \mathbb{R}^{N+1}, \\
& M=\left[\left(e_{i}, e_{j}\right)\right]_{(N+1) \times(N+1)} \in \mathbb{R}^{(N+1) \times(N+1)}, \\
& K=\left[\left(e_{i}^{\prime}, e_{j}^{\prime}\right)\right]_{(N+1) \times(N+1)} \in \mathbb{R}^{(N+1) \times(N+1)}, \\
& W=\left[\left(e_{i}, e_{j}^{\prime}\right)\right]_{(N+1) \times(N+1)} \in \mathbb{R}^{(N+1) \times(N+1)}, \\
& Q(X(t), Y(t)) \\
& =\left[\sum_{j=0}^{N} \sum_{k=0}^{N} X_{j}(t) Y_{k}(t)\left(e_{j} e_{k}, e_{i}\right)\right]_{(N+1) \times 1} \\
& =\left[X(t)^{\top} A_{i} Y(t)\right]_{(N+1) \times 1} \in \mathbb{R}^{(N+1)},
\end{aligned}
$$

where $A_{i}=\left[\left(e_{j} e_{k}, e_{i}\right)\right]_{(N+1) \times(N+1)} \in \mathbb{R}^{(N+1) \times(N+1)}, i=0,1$, $\ldots, N$.

Let

$$
\begin{aligned}
B & =\left[e_{i}(0)\right]_{(N+1) \times 1} \in \mathbb{R}^{N+1}, \\
\zeta_{1} & =\left[\left(x_{0}, e_{i}\right)\right]_{(N+1) \times 1} \in \mathbb{R}^{N+1}, \\
\zeta_{2} & =\left[\left(y_{0}, e_{i}\right)\right]_{(N+1) \times 1} \in \mathbb{R}^{N+1} .
\end{aligned}
$$

Then, (15) and (16) can be rewritten as

$$
\begin{aligned}
& M \dot{X}(t)+\beta_{1} K X(t)+v W X(t)+B u_{1}(t) \\
& \quad+r_{1} Q(X(t), Y(t))=0, \\
& M \dot{Y}(t)+\beta_{2} K Y(t)+v W Y(t)+B u_{2}(t) \\
& \quad+r_{2} Q(X(t), Y(t))=0, \\
& X(0)=M^{-1} \zeta_{1}, \\
& Y(0)=M^{-1} \zeta_{2} .
\end{aligned}
$$

Define

$$
\begin{aligned}
& Z(t)=\left(\begin{array}{l}
X(t) \\
Y(t)
\end{array}\right), \\
& R=\left(\begin{array}{cc}
-\beta_{1} M^{-1} K-v M^{-1} W & 0 \\
0 & -\beta_{2} M^{-1} K-v M^{-1} W
\end{array}\right), \\
& F=\left(\begin{array}{cc}
-M^{-1} B & 0 \\
0 & -M^{-1} B
\end{array}\right), \\
& G(Z(t))=\left(\begin{array}{l}
-r_{1} M^{-1} Q(X(t), Y(t)) \\
-r_{2} M^{-1} Q(X(t), Y(t))
\end{array}\right) .
\end{aligned}
$$

Thus, (19) is equivalent to the following equation:

$$
\begin{aligned}
& \dot{Z}(t)=H(t, Z(t), u(t)), \\
& Z(0)=Z^{0},
\end{aligned}
$$

where $H(t, Z(t), u(t))=R Z(t)+F u(t)+G(Z(t))$, and

$$
Z^{0}=\left(\begin{array}{c}
M^{-1} \zeta_{1} \\
M^{-1} \zeta_{2}
\end{array}\right)
$$

Now, we get a new time optimal control problem $\left(\mathscr{T} \mathscr{P}^{h}\right)$ governed by ordinary differential equations as follows:

$$
\max _{(u, \tau) \in \mathscr{U}}\left\{\tau \mid Z(T) \in \mathscr{K}^{h}\right\},
$$

where

$$
\begin{aligned}
\mathscr{K}^{h} & =\left\{\left(\begin{array}{l}
X \\
Y
\end{array}\right) \in \mathbb{R}^{N+1} \times \mathbb{R}^{N+1} \mid X\right. \\
& =\left(X_{1}, X_{2}, \ldots, X_{N+1}\right)^{\top}, Y \\
& =\left(Y_{1}, Y_{2}, \ldots, Y_{N+1}\right)^{\top}, \alpha_{1} \leq X_{i} \leq \gamma_{1}, \alpha_{2} \leq Y_{i} \\
& \left.\leq \gamma_{2}, i=1,2, \ldots, N+1\right\},
\end{aligned}
$$

and $Z(t)$ is the solution to (21).

\section{Control Parameterization Method and Time-Scaling Transformation}

To solve the problem $\left(\mathscr{T}^{h}\right)$ numerically, which is a time optimal control problem in the finite dimensional space, we apply the classical control parametrization method [19], where the control function can be approximated by the piecewise constant functions. We subdivide the time horizon $[0, T]$ into $p$ subintervals $\left[s_{k-1}, s_{k}\right], k=1,2, \ldots, p$, where $p \geq 2$ is a given integer and $s_{k}, k=0,1, \ldots, p$, satisfy

$$
0=s_{0} \leq s_{1}=\tau \leq s_{2} \leq \cdots \leq s_{p}=T .
$$


Here each time point $s_{k}, k=1,2, \ldots, p-1$, is called a switching time. We allow the approximate control to switch at each switching time. To find the optimal switching time points, we assume that these switching points are not prefixed and are the parameters, which need to be chosen optimally in the following set:

$$
\begin{aligned}
\Gamma & =\left\{\varsigma=\left(s_{1}, \ldots, s_{p-1}\right)^{\top} \in \mathbb{R}^{p-1} \mid 0 \leq s_{1} \leq s_{2} \leq \cdots\right. \\
& \left.\leq s_{p-1} \leq T\right\} .
\end{aligned}
$$

Define

$$
\begin{aligned}
\mathcal{U}^{p} & =\left\{\sigma=\left(\sigma^{1}, \sigma^{2}, \ldots, \sigma^{p}\right) \in \mathbb{R}^{2 \times p} \mid \sigma^{k}=\left(\sigma_{1}^{k}, \sigma_{2}^{k}\right)^{\top}\right. \\
& \left.\in \mathbb{R}^{2}, \sigma^{1}=0, a_{i} \leq \sigma_{i}^{k} \leq b_{i}, k=2, \ldots, p, i=1,2\right\} .
\end{aligned}
$$

We approximate the control function $u(t)$ by a constant vector on each subinterval:

$$
u(t) \approx \sigma^{k}, \text { for } t \in\left[s_{k-1}, s_{k}\right), k=1, \ldots, p .
$$

Then the control function can be approximated by the following piecewise constant control of the form

$$
\begin{array}{r}
u(t) \approx u^{p}(t)=\left(u_{1}^{p}(t), u_{2}^{p}(t)\right)^{\top}=\sum_{k=1}^{p} \sigma^{k} \chi_{\left[s_{k-1}, s_{k}\right)}(t), \\
t \in[0, T] .
\end{array}
$$

It is clear that $u^{p}(t)$ can switch at times $s_{k}, k=1, \ldots, p-1$. Substituting (28) into (21), we can obtain that

$$
\begin{aligned}
& \dot{Z}(t)=H(t, Z(t), 0), \quad t \in\left[0, s_{1}\right), \\
& \dot{Z}(t)=H\left(t, Z(t), \sigma^{k}\right), \\
& t \in\left[s_{k-1}, s_{k}\right), k=2, \ldots, p, \\
& Z(0)=Z^{0} .
\end{aligned}
$$

Since the switching times are unknown, (29) is not easy to solve numerically. It is also difficult to integrate (29) accurately if some of the subintervals $\left[s_{k-1}, s_{k}\right], k=1, \ldots, p$, are very short. To overcome these difficulties brought about by the unknown switching times, we make use of the timescaling transformation $[20-22]$. Let $r \in[0, p]$ be a new time variable, where $p$ is the number of the time subintervals. The time-scaling transformation between $r \in[0, p]$ and $t \in$ $[0, T]$ can be established through the following differential equation:

$$
\begin{aligned}
\frac{d t(r)}{d r} & =w(r), \quad r \in[0, p], \\
t(0) & =0, \\
t(p) & =T,
\end{aligned}
$$

where $w$ is a piecewise constant function, which is defined by

$$
w(r)=\sum_{k=1}^{p} \theta_{k} \chi_{[k-1, k)}(r), \quad r \in[0, p],
$$

where $\theta_{k}=s_{k}-s_{k-1}, k=1, \ldots, p$. Integrating (30) can yield that

$$
t(r)=\sum_{m=1}^{\lfloor r\rfloor} \theta_{m}+\theta_{\lfloor r\rfloor+1}(r-\lfloor r\rfloor), \quad r \in[0, p),
$$

where $\lfloor r\rfloor$ denotes the largest integer which is less than or equal to $r$. It follows from (32) that

$$
t(k)=\sum_{m=1}^{k} \theta_{m}=s_{k}, \quad k=0,1, \ldots, p-1 .
$$

Noting that $t(p)=T$ in (30), we have $t(p)=s_{p}$. Thus

$$
t(k)=\sum_{m=1}^{k} \theta_{m}=s_{k}, \quad k=0,1, \ldots, p .
$$

Equality (34) shows that the time-scaling transformation maps $r=k$ to the $k$ th switching time $t=s_{k}, k=1, \ldots, p-1$. Moreover, it also follows from (34) that

$$
\theta_{1}+\theta_{2}+\cdots+\theta_{p}=T
$$

By taking the approximation $\bar{u}(r) \approx \bar{u}^{p}(r)=u^{p}(t(r))=$ $\sum_{k=1}^{p} \sigma^{k} \chi_{[k-1, k)}(r)$ and making variable substitution $\bar{Z}(r)=$ $Z(t(r))$, from (29) and (30), we can derive that

$$
\begin{aligned}
& \dot{\bar{Z}}(r)=\theta_{1} \bar{H}(r, \bar{Z}(r), 0), \quad r \in[0,1), \\
& \dot{\bar{Z}}(r)=\theta_{k} \bar{H}\left(r, \bar{Z}(r), \sigma^{k}\right), \\
& r \quad r \in[k-1, k), k=2, \ldots, p, \\
& \bar{Z}(0)=Z^{0},
\end{aligned}
$$

where $\bar{H}\left(r, \bar{Z}(r), \sigma^{k}\right)=H\left(t(r), Z(t(r)), \sigma^{k}\right)$. Let

$$
\begin{aligned}
\Theta^{p} & =\left\{\theta=\left(\theta_{1}, \ldots, \theta_{p}\right)^{\top} \in \mathbb{R}^{p} \mid \theta_{i} \geq 0, i\right. \\
& \left.=1, \ldots, p, \theta_{1}+\theta_{2}+\cdots+\theta_{p}=T\right\} .
\end{aligned}
$$

We denote by $\bar{Z}(r \mid(\theta, \sigma))$ the solution to (36) corresponding to $(\theta, \sigma) \in \Theta^{p} \times \mathcal{U}^{p}$, where

$$
\bar{Z}(r \mid(\theta, \sigma))=\left(\begin{array}{l}
\bar{X}(r \mid(\theta, \sigma)) \\
\bar{Y}(r \mid(\theta, \sigma))
\end{array}\right) .
$$

Through the control parameterization method and timescaling transformation, we can translate the approximation problem $\left(\mathscr{T} \mathscr{P}^{h}\right)$ into an optimal parameter selection problem $\left(\mathscr{T} \mathscr{P}_{p}^{h}\right)$ as follows:

$$
\max _{(\theta, \sigma) \in \Theta^{p} \times \mathscr{U}^{p}}\left\{\theta_{1} \mid \bar{Z}(p \mid(\theta, \sigma)) \in \mathscr{K}^{h}\right\},
$$

where $\bar{Z}(r \mid(\theta, \sigma))$ is the solution to (36). 
(1) Input the number of the subintervals in the space interval $[0, l]: N$; Input the number of the subintervals in the new time horizon $[0, p]: p \geq 2$; Input initial value : $x_{0}, y_{0}$;

Input initial pair : $(\theta, \sigma) \in \Theta^{p} \times \mathcal{U}^{p}$;

(2) Calculate corresponding matrix $M, K, W, B$, and $A_{i}, i=0,1, \ldots, N$; Calculate initial value $\bar{Z}(0)$;

(3) Solve the optimal solution $(\theta, \sigma)$ by making use of SQP algorithm;

(4) Output the value of the optimal parameters $\left(\theta^{*}, \sigma^{*}\right)$.

Algorithm 1: Procedure of solving the problem $\left(\mathscr{T}_{p} \mathscr{P}_{p}^{h}\right)$.

\section{Solving Problem $\left(\mathscr{T} \mathscr{P}_{p}^{h}\right)$}

In the problem $\left(\mathscr{T} \mathscr{P}_{p}^{h}\right)$, both $\theta_{k}, k=1, \ldots, p$, and $\sigma^{k}, k=2$, $\ldots, p$, are decision variables, where $\theta_{k}, k=1, \ldots, p$, are the time parameters and $\sigma^{k}, k=2, \ldots, p$, are the control parameters. The problem $\left(\mathscr{T} \mathscr{P}_{p}^{h}\right)$ is essentially nonlinear mathematical programming problem and hence can be solved numerically with standard mathematical programming algorithms such as Sequential Quadratic Program algorithm. Now we present framework of solving the problem $\left(\mathscr{T}_{p}^{h}\right)$ in Algorithm 1.

We make the following finite difference scheme to solve $\bar{Z}(r \mid(\theta, \sigma)), r \in(0, p]$, which is necessary in the third step of Algorithm 1. To simplify the notation, we write $\bar{Z}(r)=$ $\bar{Z}(r \mid(\theta, \sigma))$. Firstly, we divide the time horizon $[0, p]$ into $\Lambda p$ subintervals. Let $\Delta r=1 / \Lambda$ and $r_{j}=j \Delta r, j=0,1, \ldots, \Lambda p$. We obtain that

$$
\begin{aligned}
& \bar{X}((j+1) \Delta r)=\bar{X}(j \Delta r)+\Delta r \theta_{d} M^{-1} \\
& \quad \times\left(-\beta_{1} K \bar{X}(j \Delta r)-v W \bar{X}(j \Delta r)-B \sigma_{1}^{d}\right. \\
& \left.\quad-r_{1} Q(\bar{X}(j \Delta r), \bar{Y}(j \Delta r))\right), \quad j=0,1, \ldots, \Lambda p-1, \\
& \bar{Y}((j+1) \Delta r)=\bar{Y}(j \Delta r)+\Delta r \theta_{d} M^{-1} \\
& \quad \times\left(-\beta_{1} K \bar{Y}(j \Delta r)-v W \bar{Y}(j \Delta r)-B \sigma_{2}^{d}\right. \\
& \left.\quad-r_{2} Q(\bar{X}(j \Delta r), \bar{Y}(j \Delta r))\right), \quad j=0,1, \ldots, \Lambda p-1,
\end{aligned}
$$

where $d=\lfloor j / \Lambda\rfloor+1 . \bar{X}(j \Delta r)$ and $\bar{Y}(j \Delta r)$ at $j=0$ are known from the initial condition. Therefore, the computation of (39) is straightforward.

Following the procedure of Algorithm 1, we can solve the parameter selection problem $\left(\mathscr{T}_{p}^{h}\right)$. Let $\left(\theta^{*}, \sigma^{*}\right)$ be the optimal parameter for the problem $\left(\mathscr{T}_{p}^{h}\right)$. By making use of (32), the approximation of $\left(u^{*}, \tau^{*}\right)$ and $(x, y)^{\top}$ for the problem $(\mathscr{T} \mathscr{P})$ can be obtained.

\section{Numerical Simulation and Discussion}

In this section, a numerical simulation for the problem $(\mathscr{T} \mathscr{P})$ is presented. We take

$$
\begin{aligned}
& \beta_{1}=1, \\
& \beta_{2}=1, \\
& v=1, \\
& r_{1}=0.05, \\
& r_{2}=0.1, \\
& T=1, \\
& l=1, \\
& x_{0}=\sin (\pi z), \\
& y_{0}=\frac{1}{2} \sin (\pi z),
\end{aligned}
$$

in (2). The control bounds are taken as

$$
\begin{aligned}
& a_{1}=-0.2, \\
& b_{1}=0.1, \\
& a_{2}=-0.15, \\
& b_{2}=0.15,
\end{aligned}
$$

and the target set bounds are

$$
\begin{aligned}
& \alpha_{1}=0.75 \\
& \gamma_{1}=0.9 \\
& \alpha_{2}=0.4 \\
& \gamma_{2}=0.5 .
\end{aligned}
$$


TABLE 1: Optimal time $\tau^{*}$ for different $N$ and different $p$.

\begin{tabular}{lcccccc}
\hline$p$ & & & $N$ & & 30 & 40 \\
\hline 2 & 10 & 12 & 15 & 0.3685 & 0.3686 & 0.3686 \\
5 & 0.3680 & 0.3682 & 0.3685 & 0.3686 & 0.3686 & 0.3686 \\
10 & 0.3680 & 0.3682 & 0.3685 & 0.3686 & 0.3686 & 0.3686 \\
20 & 0.3680 & 0.3682 & 0.3685 & 0.3686 & 0.3686 & 0.3686 \\
\hline
\end{tabular}

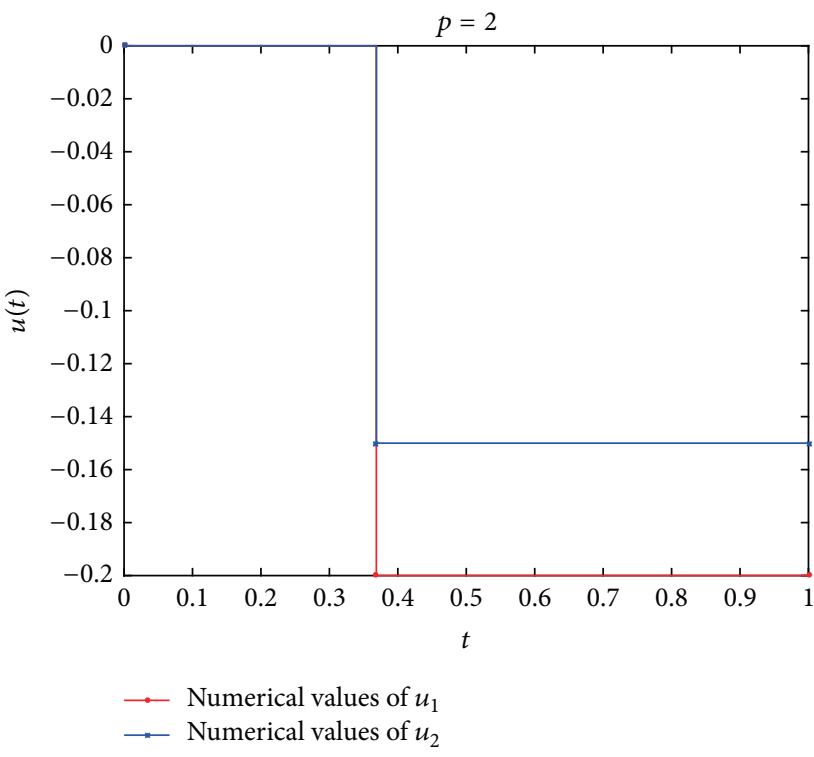

(a)

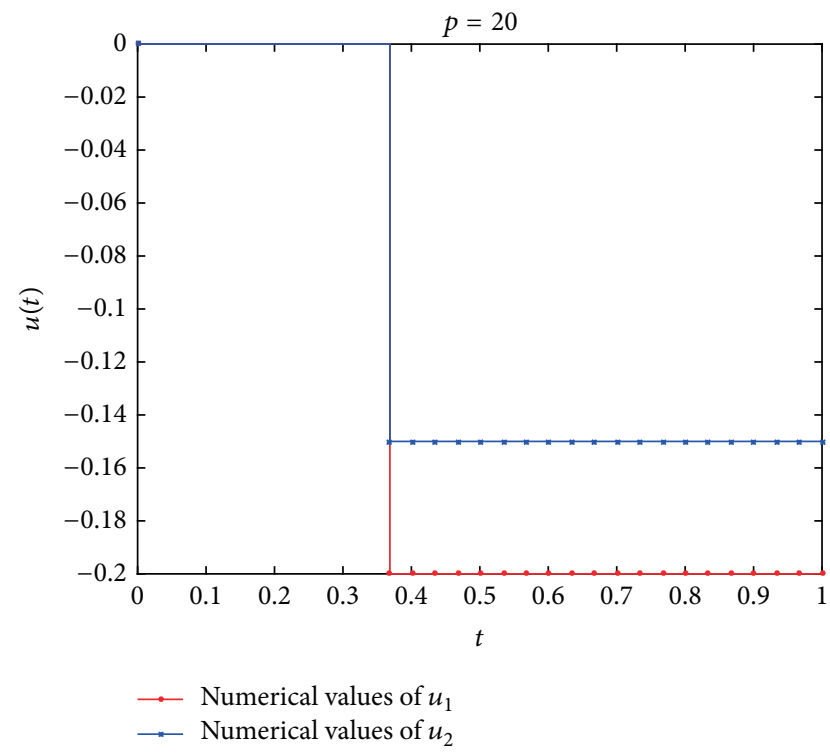

(b)

FIgURe 2: Optimal controls for different $p$.

In this simulation, we divide the interval $[0,1]$ into $N$ equal subintervals. Thus, we have $h=1 / N$. We choose the basis functions $e_{i}(z), i=0,1, \ldots, N$, as follows:

$$
\begin{aligned}
& e_{i}(z)= \begin{cases}\frac{z-z_{i-1}}{h}, & z_{i-1} \leq z<z_{i}, \\
-\frac{z-z_{i+1}}{h}, & z_{i} \leq z<z_{i+1}, \\
0, & \text { otherwise, }\end{cases} \\
& e_{0}(z)= \begin{cases}-\frac{z-z_{1}}{h}, & z_{0} \leq z<z_{1}, \\
0, & \text { otherwise, }\end{cases} \\
& e_{N}(z)= \begin{cases}\frac{z-z_{N-1},}{h}, & z_{N-1}<z \leq z_{N}, \\
0, & \text { otherwise. }\end{cases}
\end{aligned}
$$

We carry out the numerical simulation experiments within the MATLAB environment (version R2016a). We use personal computer with the following configuration: Intel Core i5-7200 2.50 GHz CPU, 8.00 GB RAM, 64-bit Windows 10 Operating System.
The following simulations are conducted by Algorithm 1. Using the piecewise constant control parameterization method with $p=2,5,10,20$, we solve problem $\left(\mathscr{T} \mathscr{P}_{p}^{h}\right)$ for $N=10,12,15,20,30,40$. The optimal time $\tau^{*}$ is given in Table 1. From Table 1, we can see that as $N$ increases from $10,12,15$ to 20 , the approximation value of $\tau^{*}$ varies from $0.3680,0.3682,0.3685$ to 0.3686 , which means finer finite element triangulation $\left(\mathscr{T}^{h}\right)$ can lead to shorter actively controlled interval $\left[\tau^{*}, T\right]$. But when $N$ increases from 20, 30 to 40 , the optimal time tends to be stable. Moreover, we see that the change of the value of $p$ has little effect on the approximation results. The optimal controls for $N=40$ when $p=2$ and $p=20$ are presented in Figure 2. It can be found in Figure 2 that the optimal control for the problem $\left(\mathscr{T P P}_{p}^{h}\right)$ is bang-bang control, and the optimal control $u^{*}$ only switches one time. The corresponding states without control and with the optimal control acting on the interval $\left(\tau^{*}, T\right)$ for $N=40$ and $p=20$ are also presented in Figures 3 and 4 , respectively.

\section{Conclusions}

In this paper, we propose a computation method for the second kind of time optimal control problem for the tubular reactors systems, which are widely used in chemical and 


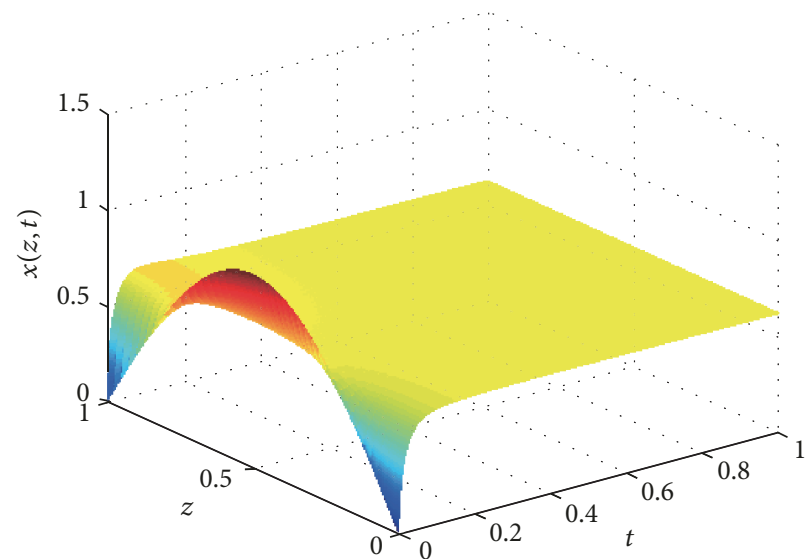

(a)

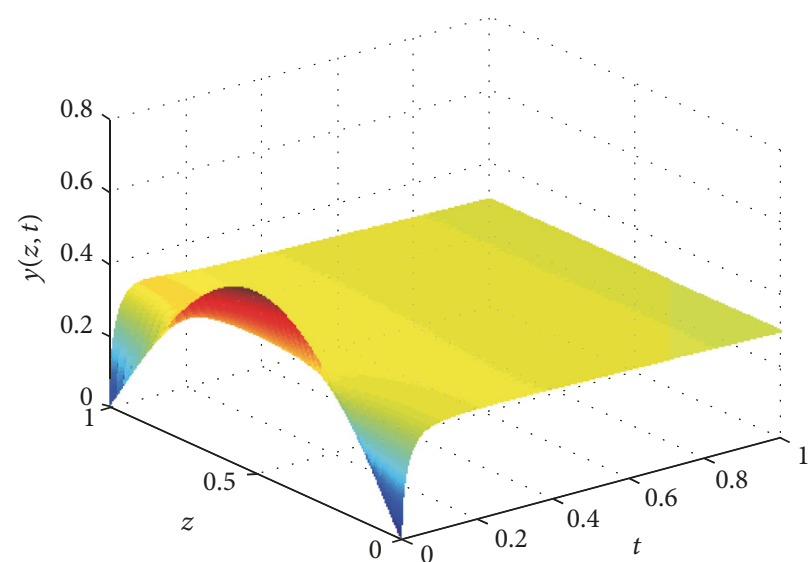

(b)

FIGURE 3: The state function without control.

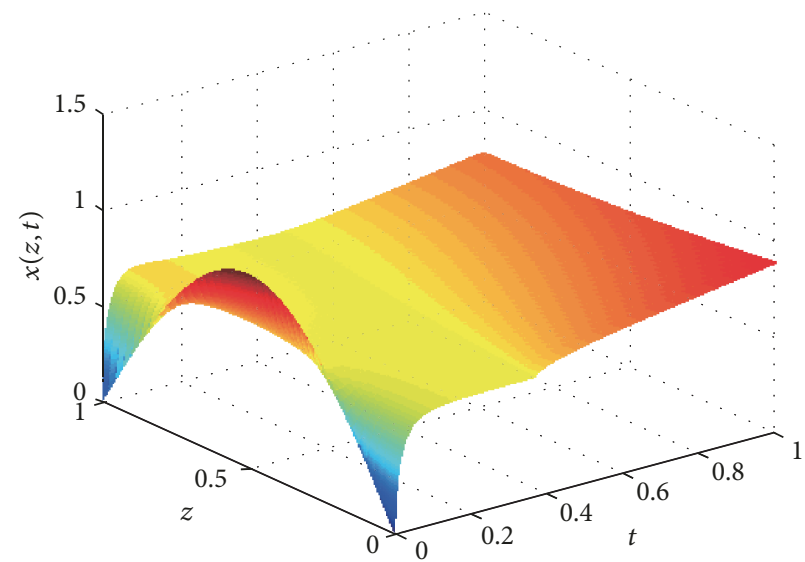

(a)

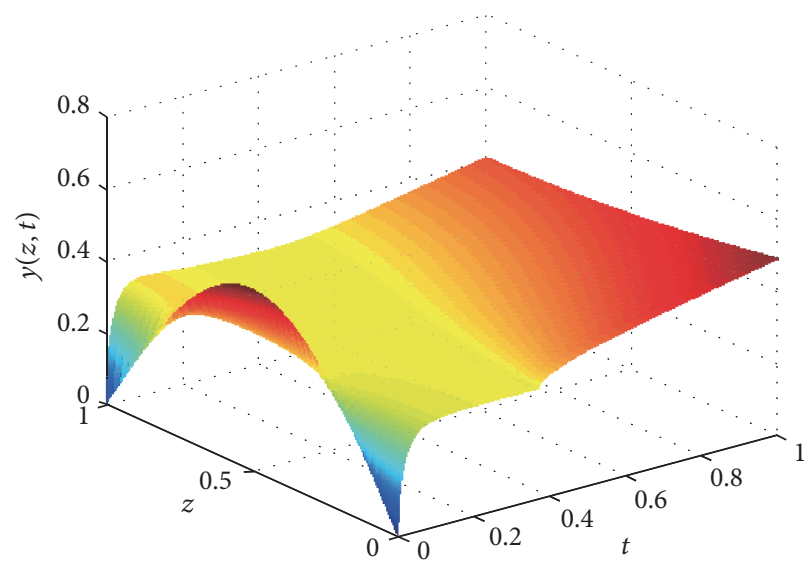

(b)

FIGURE 4: The state function with optimal control acting on the interval $\left(\tau^{*}, T\right)$.

biochemical engineering. The aim of the second kind of time optimal control problem is to find a control such that the state governed by the tubular reactors system can arrive at a given target set at the ending time $T$ with the shortest duration of the control action. Currently, the computation of the second kind of time optimal control problem did not attract enough attentions of the researchers. Our approach can be summarized in three steps: (1) A finite dimensional approximation problem can be obtained with finite element method; (2) through control parameterization method and time-scaling transformation, we translate the approximation problem into an optimal parameter selection problem; (3) we solve this optimal parameter selection problem by Sequential Quadratic Program algorithm. Moreover, we give an example for illustration to show the effectiveness of our approximation method.

\section{Conflicts of Interest}

The authors declare that there are no conflicts of interest regarding the publication of this paper.

\section{Acknowledgments}

The authors appreciate the support of the National Natural Science Foundation of China (no. 51676182, no. 61374096), the Natural Science Foundation of Zhejiang Province (no. LY17C190008), and the Natural Science Foundation of Ningbo (no. 2014A610185).

\section{References}

[1] S. Renou, M. Perrier, D. Dochain, and S. Gendron, "Solution of the convection-dispersion-reaction equation by a sequencing method," Computers \& Chemical Engineering, vol. 27, no. 5, pp. 615-629, 2003.

[2] M. Laabissi, M. E. Achhab, J. J. Winkin, and D. Dochain, "Trajectory analysis of nonisothermal tubular reactor nonlinear models," Systems \& Control Letters, vol. 42, no. 3, pp. 169-184, 2001.

[3] J. J. Winkin, D. Dochain, and P. Ligarius, "Dynamical analysis of distributed parameter tubular reactors," Automatica, vol. 36, no. 3, pp. 349-361, 2000. 
[4] B. Houska, F. Logist, J. Van Impe, and M. Diehl, "Robust optimization of nonlinear dynamic systems with application to a jacketed tubular reactor," Journal of Process Control, vol. 22, no. 6, pp. 1152-1160, 2012.

[5] N. Hudon, M. Perrier, M. Guay, and D. Dochain, "Adaptive extremum seeking control of a non-isothermal tubular reactor with unknown kinetics," Computers \& Chemical Engineering, vol. 29, no. 4, pp. 839-849, 2005.

[6] L. Mohammadi, I. Aksikas, S. Dubljevic, and J. F. Forbes, "Optimal boundary control of coupled parabolic PDE-ODE systems using infinite-dimensional representation," Journal of Process Control, vol. 33, pp. 102-111, 2015.

[7] J. Ng and S. Dubljevic, "Optimal boundary control of a diffusion-convection-reaction PDE model with time-dependent spatial domain: Czochralski crystal growth process," Chemical Engineering Science, vol. 67, no. 1, pp. 111-119, 2012.

[8] V. Barbu, Analysis and Control of Nonlinear Infinite Dimensional Systems, Academic Press, Boston, Mass, USA, 1993.

[9] X. Li and J. Yong, Optimal Control Theory for Infinite Dimensional Systems, Birkhäuser, Boston, Mass, USA, 1995.

[10] L. Wang and G. Wang, "The optimal time control of a phasefield system," SIAM Journal on Control and Optimization, vol. 42, no. 4, pp. 1483-1508, 2003.

[11] G. Wang and E. Zuazua, "On the equivalence of minimal time and minimal norm controls for internally controlled heat equations," SIAM Journal on Control and Optimization, vol. 50, no. 5, pp. 2938-2958, 2012.

[12] V. J. Mizel and T. I. Seidman, "An abstract bang-bang principle and time-optimal boundary control of the heat equation," SIAM Journal on Control and Optimization, vol. 35, no. 4, pp. 12041216, 1997.

[13] G. Wang and Y. Xu, "Equivalence of three different kinds of optimal control problems for heat equations and its applications," SIAM Journal on Control and Optimization, vol. 51, no. 2, pp. 848-880, 2013.

[14] B. Aylaj, M. E. Achhab, and M. Laabissi, "Asymptotic behaviour of state trajectories for a class of tubular reactor non-linear models," IMA Journal of Mathematical Control and Information, vol. 24, no. 2, pp. 163-175, 2007.

[15] B. Aylaj, M. E. Achhab, and M. Laabissi, "State trajectories analysis for a class of tubular reactor nonlinear nonautonomous models," Abstract and Applied Analysis, vol. 2008, Article ID 127394, 2008

[16] K. Ito and K. Kunisc, "Semismooth Newton methods for timeoptimal control for a class of ODES," SIAM Journal on Control and Optimization, vol. 48, no. 6, pp. 3997-4013, 2010.

[17] C. Y. Kaya and J. L. Noakes, "Computational method for timeoptimal switching control," Journal of Optimization Theory and Applications, vol. 117, no. 1, pp. 69-92, 2003.

[18] K. Kunisch and D. Wachsmuth, "On time optimal control of the wave equation and its numerical realization as parametric optimization problem," SIAM Journal on Control and Optimization, vol. 51, no. 2, pp. 1232-1262, 2013.

[19] K. L. Teo, C. J. Goh, and K. H. Wong, A Unified Computational Approach to Optimal Control Problems, Longman Scientific and Technical, UK, 1991.

[20] H. W. J. Lee, K. L. Teo, V. Rehbock, and L. S. Jennings, “Control parametrization enhancing technique for optimal discretevalued control problems," Automatica, vol. 35, no. 8, pp. 14011407, 1999.
[21] R. C. Loxton, K. L. Teo, V. Rehbock, and K. F. Yiu, "Optimal control problems with a continuous inequality constraint on the state and the control," Automatica, vol. 45, no. 10, pp. 2250-2257, 2009.

[22] Q. Lin, R. Loxton, K. L. Teo, and Y. H. Wu, "Optimal control computation for nonlinear systems with state-dependent stopping criteria," Automatica, vol. 48, no. 9, pp. 2116-2129, 2012. 


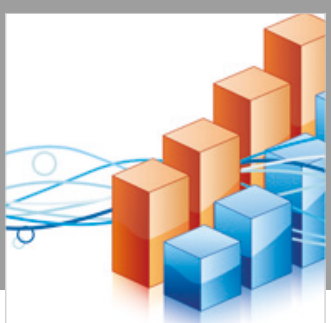

Advances in

Operations Research

\section{-n-m}
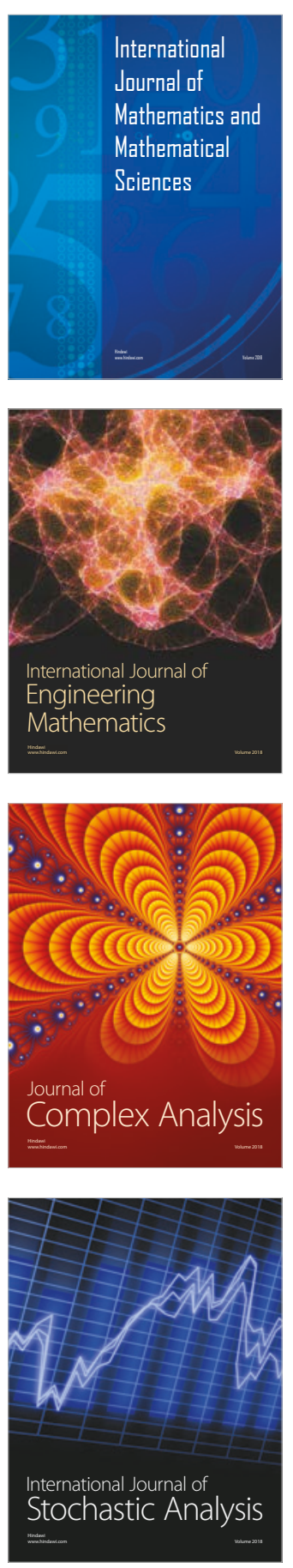
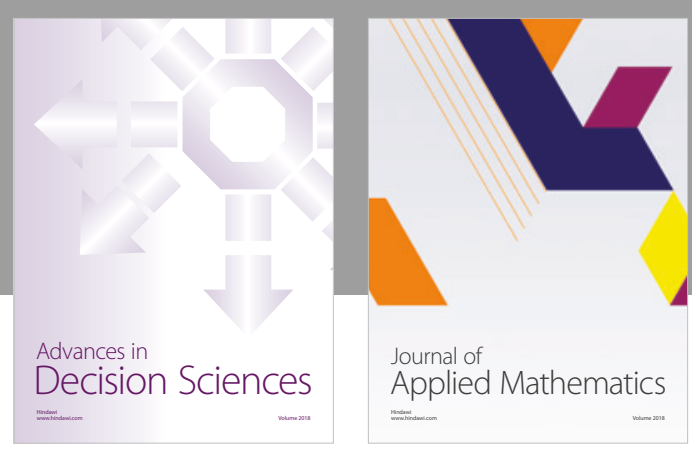

Journal of

Applied Mathematics
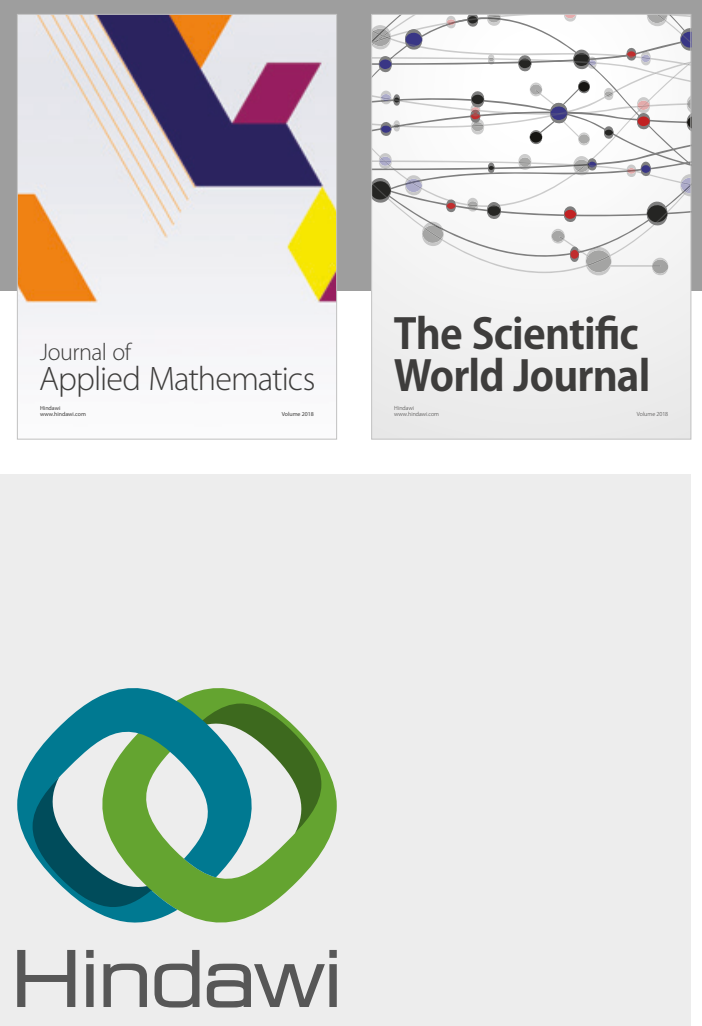

Submit your manuscripts at

www.hindawi.com

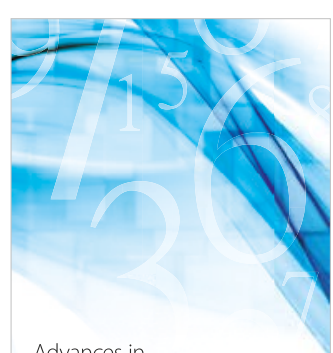

Advances in
Numerical Analysis
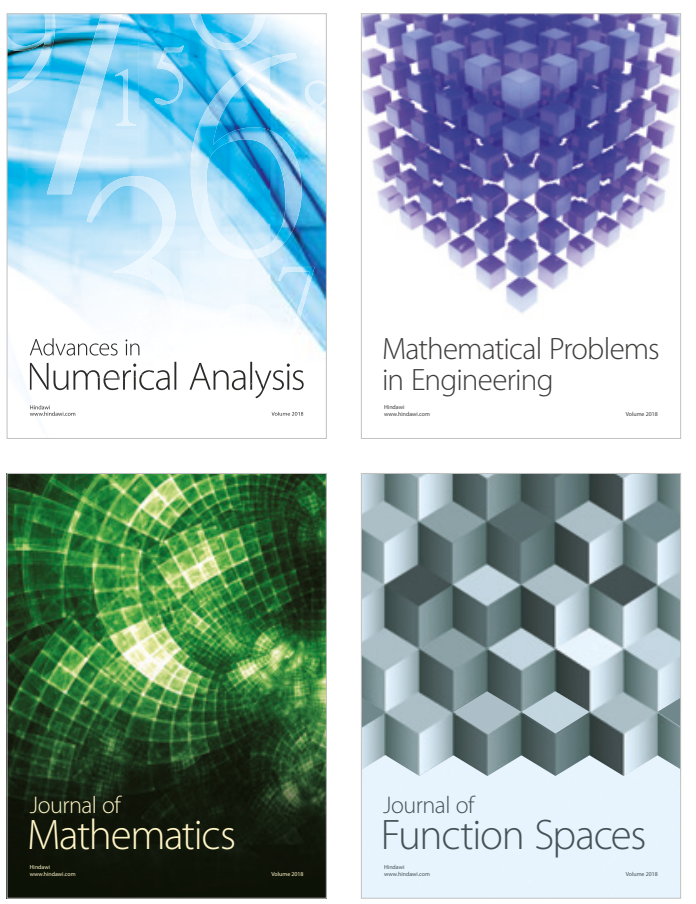

Mathematical Problems in Engineering

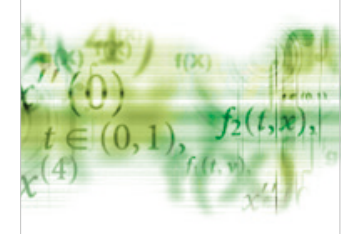

International Journal of

Differential Equations

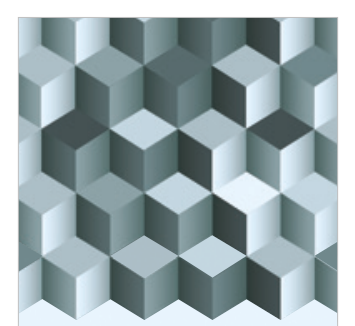

Journal of

Function Spaces

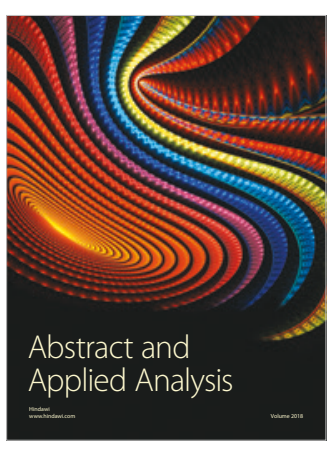

The Scientific

World Journal

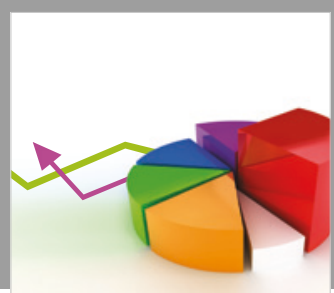

Journal of

Probability and Statistics
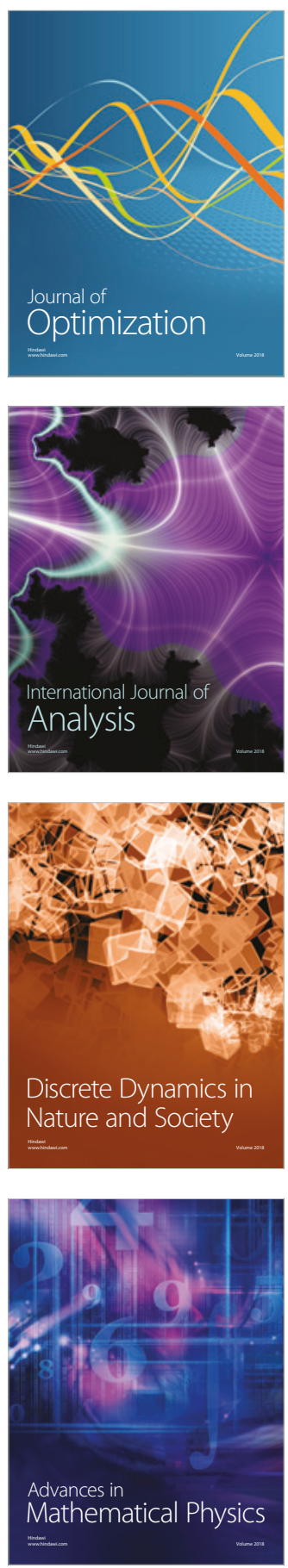